\title{
Partial Compensation of Unbalance by One- and Two-Plane Automatic Balancing Devices
}

\author{
Boris Ryzhik, Henner Duckstein, and Lutz Sperling \\ Institut für Mechanik, Otto-von-Guericke Universität Magdeburg, Magdeburg, Germany
}

This article presents an analysis of several aspects of oneand two-plane automatic balancing devices of rigid rotors. The effect of partial compensation of unbalance and the decreasing of vibrations in the region beyond the first critical speed is studied analytically and confirmed by the results of computer simulations. The possibility of partial unbalance compensation is very important considering application of autobalancing devices to rotors with a large polar moment of inertia.

Among the most important practical applications of devices are centrifuges, hand power tools, washing machines, and optical disk drives.

Keywords Autobalancing device, Rigid rotor, Synchronization, Vibrations

A rigid rotor balancing device, comprised of two balls in a circular track for compensating unbalanced inertia forces, was first introduced by Thearle (1932). Since then, autobalancing of rigid rotors by employing devices of this type has become a well-known phenomenon (see the bibliography in Blekhman (2000) and Sperling et al. (1998)). Recently, the interest in autobalancing and the number of publications on this subject has been noticeable increasing.

Some important aspects of autobalancing were considered in a number of recent publications, i.e., the papers of Chung and Ro (1999), Kang et al. (2001), and Huang and Chao (2002). In Chung and Ro (1999), the dynamic stability and the time responses have been analyzed for the automatic one-plane balancer in dependence on the system parameters. The study in

Received 22 January 2003; accepted 30 June 2003.

Address correspondence to Boris Ryzhik, Otto-von-GuerickeUniversität Magdeburg, Universitätsplatz 2, D-39106 Magdeburg, Germany. E-mail: boris.ryzhik@mb.uni-magdeburg.de
Kang et al. (2001) evaluates the performance of a ball-type balancer system that is installed in high-speed optical disk drives. The established mathematical model is analyzed by the method of multiple scales. On the basis of the possible steady-state solutions and the results of their stability analysis, general design guidelines are given. The paper of Huang and Chao (2002) is also dedicated to the design of a ball-type balancer system installed on the high-speed disk drive. After a parametric analysis, the effects of rolling resistance and drag force induced by the dynamic interaction between the ball and the fluid-filled race are considered and experimental results are discussed.

The majority of publications investigate a one-plane autobalancing device, which provides a compensation of unbalanced forces. However, for many practical applications, it is important to influence both the unbalanced force and the unbalanced moment. A device of such type was introduced by Hedaya and Sharp (1977), who propose a dynamic balancer with two circular tracks and four balls. Their paper presents a stability analysis of the balancing state together with the results of parametric studies. They also refer to experimental and simulation investigations, however without reporting any results. There are hardly any further publications about automatic balancing of both static and dynamic unbalance. However, Bövik and Högfors (1986) have investigated a sample of a non-planar rotor system also permitting axial motions of two particles in the balancing device.

The two-plane rigid rotor autobalancing device was further studied in papers by Sperling and Duckstein (2001), Sperling et al. (2001), Sperling and Ryzhik et al. (2002). These publications suggest complete differential equations of motion, linearized in the vibrational coordinates, and investigate the existence and stability of compensating phases. An analysis is carried out employing the method of direct separation of motion as proposed by Kapiza (1951), developed for many applications in vibrational mechanics by Blekhman $(1976,2000)$ and applied to some rotation vibration phenomena by Sperling et al. (1998).

Apart from analytical investigations, papers by Sperling and Duckstein (2001), Sperling et al. (2001), and Sperling and 


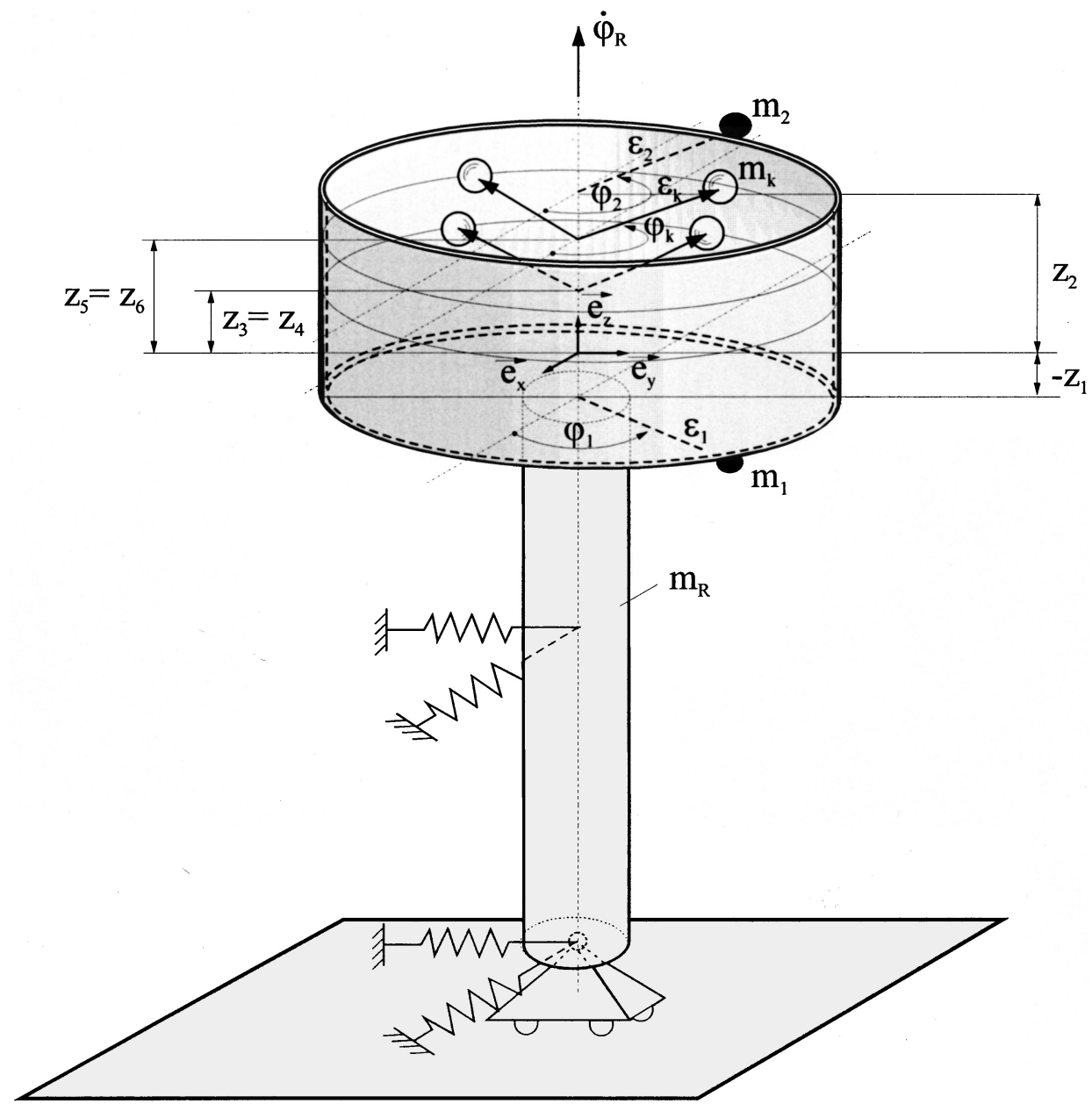

FIGURE 1

Rotor model.

Ryzhik et al. (2002) present a number of simulation results proving the possibility of stable unbalance compensation for a two-plane autobalancing device and yielding information about the influence of ball and support damping as well as rotor speed on the balancing time. These publications also show the display of Sommerfeld-type motion, whereby balls move at the speed equal to the rotor system critical speed.

The present article provides some new results concerning one- and two-plane autobalancing of rigid rotors. It investigates the effect of partial compensation of unbalance. In previous papers, the authors have proved that a stable complete compensation of unbalance is possible only for systems with the transverse moment of inertia greater than the polar one in the speed range after the second critical speed. This publication reveals that under certain conditions an autobalancing device can partially compensate unbalance and decrease vibrations in the region after the first critical speed. This compensation can be achieved even if the rotor polar moment of inertia exceeds the transverse moment.

\section{MODEL AND EQUATIONS OF MOTION}

Figure 1 shows a rigid rotor with a two-plane autobalancing device (see Sperling et al. $(2001,2002)$ ). The axial symmetric rotor has a mass $m_{R}$ and moments of inertia $J_{x x R}=J_{y y R}=J_{a R}$, $J_{z z R}=J_{z R}, J_{x y R}=J_{y z R}=J_{z x R}=0$ with respect to the center of mass in the non-rotating vector frame $\vec{e}_{x}, \vec{e}_{y}, \vec{e}_{z}$. It also has two unbalances in the planes $z_{1}, z_{2}$, in the following called primary unbalances, idealized as mass points with the masses $m_{1}, m_{2}$ and eccentricities $\varepsilon_{1}, \varepsilon_{2}$. The rotor axial angular velocity is $\dot{\varphi}_{R}$, $\varphi_{i}=\varphi_{R}+\gamma_{i}, i=1,2$ are the angular positions of the primary unbalances, and $\mathbf{q}_{V}=\left[\begin{array}{llll}x & y & \psi_{x} & \psi_{y}\end{array}\right]^{T}$ are the coordinates of the vibrational motion (see Figure 2).

The angular coordinates $\varphi_{i}(i=3, \ldots, 6)$ describe the motion of the four compensation elements of the autobalancing device (see Figure 1), characterized by the mass, eccentricity of the mass center, radius, position of the plane $\left(m_{i}, \varepsilon_{I}, r_{i}, z_{i}, i=3, \ldots\right.$, 6 , respectively), and moments of inertia $J_{x x i}=J_{y y i}=J_{q i}$, $J_{z z i}=J_{p i}, J_{x y i}=J_{y z i}=J_{z x i}=0$ with respect to the center of mass. It is assumed that the elements roll along the tracks 

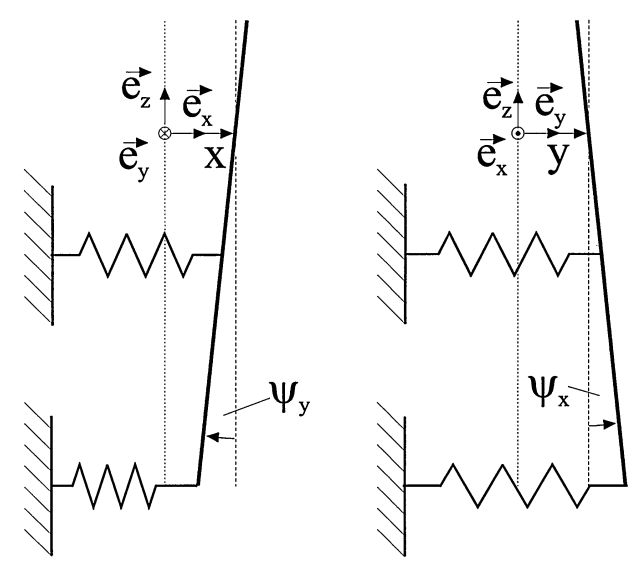

FIGURE 2

Main system variables.

without slipping and viscous medium exerts a damping moment on them.

Also considering external damping, the overall damping moment acting upon the rotor is (see Sperling et al. $(2001,2002)$ )

$$
\begin{aligned}
M_{d} & =-\left[\bar{\beta}_{R} \dot{\varphi}_{R}+\sum_{i=3}^{6} \beta_{i}\left(\dot{\varphi}_{R}-\dot{\varphi}_{i}\right)\right]=-\beta_{R} \dot{\varphi}_{R}+\sum_{i=3}^{6} \beta_{i} \dot{\varphi}_{i}, \\
\beta_{R} & =\bar{\beta}_{R}+\sum_{i=3}^{6} \beta_{i} .
\end{aligned}
$$

Using the abbreviations

$$
\begin{aligned}
M & =m_{R}+\sum_{i=1}^{6} m_{i}, \quad J_{z}=J_{z R}+\sum_{i=3}^{6}\left(\frac{R_{i}}{r_{i}}\right)^{2} J_{p i}, \\
\tilde{J}_{z} & =J_{z R}+\sum_{i=3}^{6} \frac{R_{i}}{r_{i}} J_{p i}, \quad R_{i}=r_{i}+\varepsilon_{i} \\
J_{i} & =\tilde{J}_{i}=m_{i} \varepsilon_{i}^{2}, \quad i=1,2, \quad J_{i}=m_{i} \varepsilon_{i}^{2}+\left(\frac{\varepsilon_{i}}{r_{i}}\right)^{2} J_{p i}, \\
\tilde{J}_{i} & =m_{i} \varepsilon_{i}^{2}-\frac{\varepsilon_{i}}{r_{i}} J_{p i}, \quad i=3, \ldots, 6, \\
J_{i R} & =\frac{R_{i} \varepsilon_{i}}{r_{i}^{2}} J_{p i}, \quad J_{a}=J_{a R}+\sum_{i=1}^{6} m_{i} z_{i}^{2}+\sum_{i=3}^{6} J_{q i}, \\
i & =3, \ldots, 6,
\end{aligned}
$$

we obtain the following Lagrange's equations for the system under investigation, linearized in the vibrational coordinates $\mathbf{q}_{V}$ (see Sperling et al., 2001, 2002):

$$
\begin{aligned}
& M \ddot{x}+\sum_{k=1}^{6} m_{k} z_{k} \ddot{\psi}_{y}+c_{11} \dot{x}+c_{12} \dot{\psi}_{y}+k_{11} x+k_{12} \psi_{y} \\
& =\sum_{k=1}^{6} m_{k} \varepsilon_{k}\left(\ddot{\varphi}_{k} \sin \varphi_{k}+\dot{\varphi}_{k}^{2} \cos \varphi_{k}\right),
\end{aligned}
$$

$$
\begin{aligned}
& M \ddot{y}-\sum_{k=1}^{6} m_{k} z_{k} \ddot{\psi}_{x}+c_{11} \dot{y}-c_{12} \dot{\psi}_{x}+k_{11} y-k_{12} \psi_{x} \\
& =-\sum_{k=1}^{6} m_{k} \varepsilon_{k}\left(\ddot{\varphi}_{k} \cos \varphi_{k}-\dot{\varphi}_{k}^{2} \sin \varphi_{k}\right), \\
& -\sum_{k=1}^{6} m_{k} z_{k} \ddot{y}+J_{a} \ddot{\psi}_{x}+\frac{1}{2} \sum_{k=1}^{6} m_{k} \varepsilon_{k}^{2}\left[\ddot{\psi}_{x}\left(1-\cos 2 \varphi_{k}\right)\right. \\
& \left.-\ddot{\psi}_{y} \sin 2 \varphi_{k}\right] \\
& -c_{12} \dot{y}+c_{22} \dot{\psi}_{x}+\left[\tilde{J}_{z} \dot{\varphi}_{R}+\sum_{k=1}^{6} \tilde{J}_{k} \dot{\varphi}_{k}\right] \dot{\psi}_{y} \\
& +\sum_{k=1}^{6} m_{k} \varepsilon_{k}^{2} \dot{\varphi}_{k}\left(\dot{\psi}_{x} \sin 2 \varphi_{k}-\dot{\psi}_{y} \cos 2 \varphi_{k}\right) \\
& -k_{12} y+k_{22} \psi_{x}+\frac{1}{2}\left[\tilde{J}_{z} \ddot{\varphi}_{R}+\sum_{i=1}^{6} \tilde{J}_{k} \ddot{\varphi}_{k}\right] \psi_{y} \\
& =\sum_{k=1}^{6} m_{k} \varepsilon_{k} z_{k}\left(\ddot{\varphi}_{k} \cos \varphi_{k}-\dot{\varphi}_{k}^{2} \sin \varphi_{k}\right), \\
& \sum_{k=1}^{6} m_{k} z_{k} \ddot{x}+J_{a} \ddot{\psi}_{y}+\frac{1}{2} \sum_{k=1}^{6} m_{k} \varepsilon_{k}^{2}\left[-\ddot{\psi}_{x} \sin 2 \varphi_{k}\right. \\
& \left.+\ddot{\psi}_{y}\left(1+\cos 2 \varphi_{k}\right)\right]+c_{12} \dot{x}+c_{22} \dot{\psi}_{y}-\left[\tilde{J}_{z} \dot{\varphi}_{R}+\sum_{k=1}^{6} \tilde{J}_{k} \dot{\varphi}_{k}\right] \dot{\psi}_{x} \\
& -\sum_{k=1}^{6} m_{k} \varepsilon_{k}^{2} \dot{\varphi}_{k}\left(\dot{\psi}_{x} \cos 2 \varphi_{k}+\dot{\psi}_{y} \sin 2 \varphi_{k}\right) \\
& +k_{12} x+k_{22} \psi_{y}-\frac{1}{2}\left[\tilde{J}_{z} \ddot{\varphi}_{R}+\sum_{k=1}^{6} \tilde{J}_{k} \ddot{\varphi}_{k}\right] \psi_{x} \\
& =\sum_{k=1}^{6} m_{k} \varepsilon_{k} z_{k}\left(\ddot{\varphi}_{k} \sin \varphi_{k}+\dot{\varphi}_{k}^{2} \cos \varphi_{k}\right) \text {, } \\
& \left(J_{z}+\sum_{k=1}^{2} J_{k}\right) \ddot{\varphi}_{R}-\sum_{k=3}^{6} J_{k R} \ddot{\varphi}_{k}+\beta_{R} \dot{\varphi}_{R}-\sum_{i=3}^{6} \beta_{k} \dot{\varphi}_{k} \\
& -\sum_{k=1}^{2} m_{k} \varepsilon_{k}\left[\left(\ddot{x}+z_{k} \ddot{\psi}_{y}\right) \sin \varphi_{k}+\left(-\ddot{y}+z_{k} \ddot{\psi}_{x}\right) \cos \varphi_{k}\right] \\
& =L_{R}\left(\dot{\varphi}_{R}\right) \text {, } \\
& -J_{i R} \ddot{\varphi}_{R}+J_{i} \ddot{\varphi}_{i}-\beta_{i} \dot{\varphi}_{R}+\beta_{i} \dot{\varphi}_{i}-m_{i} \varepsilon_{i}\left[\left(\ddot{x}+z_{i} \ddot{\psi}_{y}\right) \sin \varphi_{i}\right. \\
& \left.-\left(\ddot{y}-z_{i} \ddot{\psi}_{x}\right) \cos \varphi_{i}\right]=0, \quad i=3, \ldots, 6
\end{aligned}
$$

The isotropic elastic damping supports of the rotor are characterized by the stiffnesses $k_{11}, k_{12}, k_{22}$ and damping parameters $c_{11}, c_{12}, c_{22}$ with respect to the vibrational co-ordinates $\mathbf{q}_{V}$. $L_{R}=L_{R}\left(\dot{\varphi}_{R}\right)$ is the rotor driving torque.

This complete system of equations of motion is used in computer simulations.

\section{GENERAL EXISTENCE AND STABILITY CONDITIONS OF SYNCHRONOUS MOTIONS}

The method of direct separation of motion as suggested by Kapiza (1951) and advanced and frequently applied by 
Blekhman $(1976,2000)$ has proven to be a successful tool for investigating synchronization problems. Its application to the present analysis is fairly simple due to the system isotropy.

In the following, the analytical investigation is proceeded under the assumption of a constant rotor angular velocity: $\dot{\varphi}_{R}=$ $\Omega=$ const., $\varphi_{R}=\Omega t$.

Following the method of direct separation, of motion we suppose $\varphi_{i}(t)=\Omega t+\alpha_{i}(t), i=3, \ldots, 6$. Thus, the Equation (8) yields

$$
J_{i} \ddot{\alpha}_{i}+\beta_{i} \dot{\alpha}_{i}+V_{i}=0 \quad i=3, \ldots, 6
$$

with the so-called vibrational moments

$$
\begin{aligned}
V_{i}= & -m_{i} \varepsilon_{i}\left[\left(\ddot{x}+z_{i} \ddot{\psi}_{y}\right) \sin \left(\Omega t+\alpha_{i}\right)\right. \\
& \left.-\left(\ddot{y}-z_{i} \ddot{\psi}_{x}\right) \cos \left(\Omega t+\alpha_{i}\right)\right], \quad i=3, \ldots, 6 .
\end{aligned}
$$

The synchronous motion is specified by solutions of Equation (9) in the form $\alpha_{i}(t)=\alpha_{i}^{*}=$ const., $i=3, \ldots, 6$. Thus, we obtain from (9) and (10) the following existence conditions of synchronous motion:

$$
\begin{aligned}
V_{i}^{0}= & -m_{i} \varepsilon_{i}\left[\left(\ddot{x}^{0}+z_{i} \ddot{\psi}_{y}^{0}\right) \sin \left(\Omega t+\alpha_{i}^{*}\right)\right. \\
& \left.-\left(\ddot{y}^{0}-z_{i} \ddot{\psi}_{x}^{0}\right) \cos \left(\Omega t+\alpha_{i}^{*}\right)\right]=0, \quad i=3, \ldots, 6 .
\end{aligned}
$$

The vibrational moments $V_{1}^{0}$ are caused by the corresponding rotor vibrations and retroact on the compensation elements. The expressions in parentheses represent accelerations of the circular path centers of the elements and need to be determined for the stationary motion using Equations (3)-(6).

Neglecting the unbalance masses and moments of inertia as well as any damping terms and considering that $\varphi_{i}=\Omega t+\alpha_{i}^{*}$, $i=1, \ldots, 6, \alpha_{i}^{*}=\gamma_{i}, i=1,2$, we obtain

$$
\begin{aligned}
& M \ddot{x}^{0}+k_{11} x^{0}+k_{12} \psi_{y}^{0}=\sum_{k=1}^{6} f_{k} \cos \left(\Omega t+\alpha_{k}^{*}\right), \\
& J_{a} \ddot{\psi}_{x}^{0}+J_{z} \Omega \dot{\psi}_{y}^{0}-k_{12} y^{0}+k_{22} \psi_{x}^{0}=-\sum_{k=1}^{6} z_{k} f_{k} \sin \left(\Omega t+\alpha_{k}^{*}\right) \\
& M \ddot{y}^{0}+k_{11} y^{0}-k_{12} \psi_{x}^{0}=\sum_{k=1}^{6} f_{k} \sin \left(\Omega t+\alpha_{k}^{*}\right), \\
& J_{a} \ddot{\psi}_{y}^{0}-J_{z} \Omega \dot{\psi}_{x}^{0}+k_{12} x^{0}+k_{22} \psi_{y}^{0}=\sum_{k=1}^{6} z_{k} f_{k} \cos \left(\Omega t+\alpha_{k}^{*}\right)
\end{aligned}
$$

with the centrifugal forces $f_{k}=m_{k} \varepsilon_{k} \Omega^{2}$.

The stationary solution of these equations yields

$$
\begin{aligned}
& r_{i x}^{0}=x^{0}+z_{i} \psi_{y}^{0}=\sum_{k=1}^{6} f_{k} A_{i k} \cos \left(\Omega t+\alpha_{k}^{*}\right), \\
& r_{i y}^{0}=y^{0}-z_{i} \psi_{x}^{0}=\sum_{k=1}^{6} f_{k} A_{i k} \sin \left(\Omega t+\alpha_{k}^{*}\right)
\end{aligned}
$$

with

$$
\begin{aligned}
A_{i k}= & \frac{1}{\Delta}\left\{-\left(J_{a}-J_{z}\right) \Omega^{2}+k_{22}-k_{12} z_{k}\right. \\
& \left.+z_{i}\left[\left(-M \Omega^{2}+k_{11}\right) z_{k}-k_{12}\right]\right\}, \\
\Delta= & M\left(J_{a}-J_{z}\right) \Omega^{4}-\left[M k_{22}+\left(J_{a}-J_{z}\right) k_{11}\right] \Omega^{2} \\
& +k_{11} k_{22}-k_{12}^{2} .
\end{aligned}
$$

From Equations (11) and (14), we obtain the following simple existence conditions for determining the phases of synchronous motions:

$$
\begin{aligned}
V_{i}^{0} & =V_{i}^{0}\left(\alpha_{1}^{*}, \ldots, \alpha_{6}^{*}\right)=f_{i} \sum_{k=1}^{6} f_{k} A_{i k} \sin \left(\alpha_{i}^{*}-\alpha_{k}^{*}\right)=0 \\
i & =3, \ldots, 6
\end{aligned}
$$

It should be noticed that Equation (17) yields several solutions of different types.

For investigating stability we introduce the variations $\bar{\alpha}_{i}$ by $\alpha_{i}=\alpha_{i}^{*}+\bar{\alpha}_{i}, i=1, \ldots, 6, \bar{\alpha}_{i}=0, i=1,2$ and obtain the equations

$$
\begin{gathered}
J_{i} \ddot{\bar{\alpha}}_{i}+\beta_{i} \dot{\bar{\alpha}}_{i}+f_{i} \sum_{\substack{k=1 \\
k \neq i}}^{6} f_{k} A_{i k} \cos \left(\alpha_{i}^{*}-\alpha_{k}^{*}\right)\left(\bar{\alpha}_{i}-\bar{\alpha}_{k}\right)=0 \\
i=3, \ldots, 6 .
\end{gathered}
$$

In practice it always holds true that $\beta_{i} \neq 0, i=3, \ldots, 6$, hence, necessary and sufficient condition for asymptotic stability requires the "stiffness matrix" as under Equation (18) be positively definite. This stability analysis is restricted to undamped rotor vibrations. The stability of vibrational motion can be ensured by providing proper damping.

The permissibility of such approach was confirmed by results of computer simulations. The speed diapasons of stable motions, predicted by analytical consideration and by simulations, in which the value of damping was varied in a wide range, always coincide.

\section{SPECIAL CASES}

\section{Complete Unbalance Compensation}

The alternative form of Equation (17)

$$
\begin{aligned}
V_{i}^{0}= & f_{i} A_{i R}\left[\left(\sum_{k=1}^{6} f_{k} \cos \alpha_{k}^{*}\right) \sin \alpha_{i}^{*}-\left(\sum_{k=1}^{6} f_{k} \sin \alpha_{k}^{*}\right) \cos \alpha_{i}^{*}\right] \\
& +f_{i} A_{i T}\left[\left(\sum_{k=1}^{6} f_{k} z_{k} \cos \alpha_{k}^{*}\right) \sin \alpha_{i}^{*}\right. \\
& \left.-\left(\sum_{k=1}^{6} f_{k} z_{k} \sin \alpha_{k}^{*}\right) \cos \alpha_{i}^{*}\right]=0, \quad i=3, \ldots, 6
\end{aligned}
$$


with

$$
\begin{aligned}
& A_{i k}=A_{i R}+A_{i T} z_{k}, \\
& A_{i R}=\frac{1}{\Delta}\left[-\left(J_{a}-J_{z}\right) \Omega^{2}+k_{22}-k_{12} z_{i}\right], \\
& A_{i T}=\frac{1}{\Delta}\left[\left(-M \Omega^{2}+k_{11}\right) z_{i}-k_{12}\right]
\end{aligned}
$$

shows that vanishing of the four expressions in parentheses under [19] is identical with the equilibrium conditions for the total compensation of centrifugal forces, i.e., it corresponds to complete balancing. Thus, total compensation phasing is always a solution of Equation (17).

Vanishing the parentheses in (19) simplifies Equation (18) to

$$
\begin{gathered}
J_{i} \ddot{\bar{\alpha}}_{i}+\beta_{i} \dot{\bar{\alpha}}_{i}-f_{i} \sum_{k=3}^{6} f_{k} A_{i k} \cos \left(\alpha_{i}^{*}-\alpha_{k}^{*}\right) \bar{\alpha}_{k}=0, \\
i=3, \ldots, 6 .
\end{gathered}
$$

This yields the necessary and sufficient condition for the asymptotic stability of complete compensation phasing

$$
\overline{\mathbf{A}}=\left\{-A_{i k} \cos \left(\alpha_{i}^{*}-\alpha_{k}^{*}\right)\right\}>\mathbf{0}, \quad(\overline{\mathbf{A}} \text { is positively definite }) .
$$

For a two-plane autobalancing device $z_{3}=z_{4}, z_{5}=z_{6}$. As shown in (Sperling et al., 2001), stable compensation phasing in this case is only possible in the frequency range after the second critical speed (postcritical region) and when condition $J_{a}>J_{z}$ is fulfilled. These results are confirmed by computer simulations (see Sperling et al., 2001, 2002).

\section{Partial Unbalance Compensation}

An analysis of solutions of Equation (19), corroborated by the results of simulations, shows that besides the total compensation in the postcritical region, an autobalancing device can provide "partial compensation" of unbalanced forces in the frequency range after the first critical speed. Under certain conditions, rolls take positions such as to decrease unbalance and vibrations of the rotor. This partial compensation may take place even if the rotor polar moment of inertia exceeds the transverse one. Partial compensation is very important with regard to the application of autobalancing devices to rotor systems with nominal speeds in the frequency range after the first and before the second critical speed. This class of rotors includes, for example, sufficiently unsymmetrical rotor systems with a large polar moment of inertia, such as centrifugal rotors. The first critical speed of such rotors is very low, whereas their second critical speed is high; in the case when $J_{a}<J_{z}$, they do not exhibit second critical speed at all.

\section{Partial Compensation by a One-Plane Device}

First, we investigate the effect of partial compensation considering a one-plane autobalancing device comprising two rolls.
For simplification:

$$
\begin{aligned}
& J_{3}=J_{4}=J, \quad \beta_{3}=\beta_{4}=\beta, \\
& f_{3}=f_{4}=f, \quad \mu_{i}=\frac{f_{i}}{f}, \quad i=1,2 .
\end{aligned}
$$

For a one-plane device, $z_{3}=z_{4}$, thus $A_{34}=A_{43}=A_{3}$. The existence conditions (17) in this case yield

$$
\begin{aligned}
& K_{3} \sin \left(\alpha_{3}^{*}-\psi_{3}\right)+A_{3} \sin \left(\alpha_{3}^{*}-\alpha_{4}^{*}\right)=0, \\
& K_{3} \sin \left(\alpha_{4}^{*}-\psi_{3}\right)-A_{3} \sin \left(\alpha_{3}^{*}-\alpha_{4}^{*}\right)=0,
\end{aligned}
$$

where $K_{3}, \psi_{3}$ are determined by the equations

$$
\begin{gathered}
K_{3} \cos \psi_{3}=\mu_{1} A_{31} \cos \alpha_{1}+\mu_{2} A_{32} \cos \alpha_{2}, \\
K_{3} \sin \psi_{3}=\mu_{1} A_{31} \sin \alpha_{1}+\mu_{2} A_{32} \sin \alpha_{2} .
\end{gathered}
$$

Under the condition $\left|K_{3}\right| \leq 2\left|A_{3}\right|$ Equation (24) has a solution of the type

$$
\begin{gathered}
\alpha_{3}^{*}=\psi_{3}+\pi-\gamma, \quad \alpha_{4}^{*}=\psi_{3}+\pi+\gamma \\
\text { with } \quad \gamma=\arccos \frac{K_{3}}{2 A_{3}} .
\end{gathered}
$$

Although complete compensation is impossible in this case, an analysis shows that an autobalancing device with a properly chosen plane can partially compensate unbalanced forces, providing decrease of vibrations. It is interesting that, due to (14) and (24) with (26),

$$
r_{3 x}^{0} \equiv 0, \quad r_{3 y}^{0} \equiv 0
$$

Rolls seek positions causing vibrations in the plane of the device to be equal zero. As to stability investigations, instead of (18) we obtain

$$
\begin{aligned}
J \ddot{\bar{\alpha}}_{i} & +\beta \dot{\bar{\alpha}}_{i}+f^{2}\left[K_{3} \cos \left(\alpha_{i}^{*}-\psi_{3}\right) \bar{\alpha}_{i}\right. \\
& \left. \pm A_{3} \cos \left(\alpha_{3}^{*}-\alpha_{4}^{*}\right)\left(\bar{\alpha}_{3}-\bar{\alpha}_{4}\right)\right]=0, \quad i=3,4
\end{aligned}
$$

For the solution of type (26), $\sin \left(\alpha_{3}^{*}-\alpha_{4}^{*}\right) \neq 0$; hence, the determinant of the "stiffness matrix" of Equation (28) is always positive. For the stability of phase positions the condition

$$
\begin{aligned}
-A_{3}= & -\frac{1}{\Delta}\left\{-\left(J_{a}-J_{z}\right) \Omega^{2}+k_{22}\right. \\
& \left.+\left(-M \Omega^{2}+k_{11}\right) z_{3}^{2}-2 k_{12} z_{3}\right\}>0
\end{aligned}
$$

is necessary and sufficient.

In particular, the phases are unstable under the condition $A_{3}=0$. In this case, the autobalancing device does not exert any influence on vibrations in the plane of the device. Corresponding rotational speeds are called "insensitive" in the balancing literature. Simulations show that synchronization of the rolls is disturbed at such speeds. 
In the special case of only one primary unbalance and autobalancing device in the same plane, condition (29) with (16) is necessary and sufficient for complete compensation.

\section{Partial Two-Plane Compensation}

Simulations show that in the case of a two-plane balancing with $z_{3}=z_{4}, z_{5}=z_{6}$ within frequency ranges before the highest critical speed, rolls as a rule synchronize by pairs:

$$
\alpha_{4}^{*}=\alpha_{3}^{*}, \quad \alpha_{6}^{*}=\alpha_{5}^{*} .
$$

Different types of motion may occur only if two autobalancing planes are very close to each other; however, this case is not considered in the present article.

Regardless of any other solutions that might be possible, we focus on stable "self-coupling" in terms of (30). We also assume that

$$
\begin{array}{ll}
J_{3}=\cdots=J_{6}=J, & \beta_{3}=\cdots=\beta_{6}=\beta, \\
f_{3}=\cdots=f_{6}=f ; & \mu_{i}=\frac{f_{i}}{2 f}, \quad i=1,2 .
\end{array}
$$

Thus, the existence conditions (17) yield

$$
\begin{aligned}
& K_{3} \sin \left(\alpha_{3}^{*}-\psi_{3}\right)+A_{35} \sin \left(\alpha_{3}^{*}-\alpha_{5}^{*}\right)=0, \\
& K_{5} \sin \left(\alpha_{5}^{*}-\psi_{5}\right)-A_{35} \sin \left(\alpha_{3}^{*}-\alpha_{5}^{*}\right)=0
\end{aligned}
$$

where $K_{i}, \psi_{i}, i=3,5$ are determined by the equations

$$
\begin{aligned}
K_{i} \cos \psi_{i} & =\mu_{1} A_{i 1} \cos \alpha_{1}+\mu_{2} A_{i 2} \cos \alpha_{2}, \\
K_{i} \sin \psi_{i} & =\mu_{1} A_{i 1} \sin \alpha_{1}+\mu_{2} A_{i 2} \sin \alpha_{2} .
\end{aligned}
$$

For investigating the stability we have the equations

$$
\begin{aligned}
& J \ddot{\bar{\alpha}}_{3}+\beta \dot{\bar{\alpha}}_{3}+2 f^{2}\left[K_{3} \cos \left(\alpha_{3}^{*}-\psi_{3}\right) \bar{\alpha}_{3}\right. \\
& \left.\quad+A_{35} \cos \left(\alpha_{3}^{*}-\alpha_{5}^{*}\right)\left(\bar{\alpha}_{3}-\bar{\alpha}_{5}\right)\right]=0, \\
& J \ddot{\bar{\alpha}}_{5}+\beta \dot{\bar{\alpha}}_{5}+2 f^{2}\left[K_{5} \cos \left(\alpha_{5}^{*}-\psi_{5}\right)\right. \\
& \left.\quad \bar{\alpha}_{5}-A_{35} \cos \left(\alpha_{3}^{*}-\alpha_{5}^{*}\right)\left(\bar{\alpha}_{3}-\bar{\alpha}_{5}\right)\right]=0 .
\end{aligned}
$$

If $\beta \neq 0$, the necessary and sufficient conditions for positive definiteness of the corresponding "stiffness matrix," i.e., for the asymptotic stability of the phase positions of the rolls, are

$$
\begin{aligned}
& K_{3} \cos \left(\alpha_{3}^{*}-\psi_{3}\right)+A_{35} \cos \left(\alpha_{3}^{*}-\alpha_{5}^{*}\right)>0, \\
& K_{3} K_{5} \cos \left(\alpha_{3}^{*}-\psi_{3}\right) \cos \left(\alpha_{5}^{*}-\psi_{5}\right)+\left[K_{3} \cos \left(\alpha_{3}^{*}-\psi_{3}\right)\right. \\
& \left.\quad+K_{5} \cos \left(\alpha_{5}^{*}-\psi_{5}\right)\right] A_{35} \cos \left(\alpha_{3}^{*}-\alpha_{5}^{*}\right)>0 .
\end{aligned}
$$

\section{SIMULATIONS}

The effect of partial unbalance compensation was verified by computer simulations. Simulations were performed by employing the Advanced Continuous Simulation Language (ACSL) for a model rotor with a mass $5 \mathrm{~kg}$, moments of inertia $J_{a}=$ $0.025 \mathrm{kgm}^{2}, J_{z}=0.034 \mathrm{~kg} \mathrm{~m}^{2}$, and the first critical speed $\Omega_{1}=26.4 \mathrm{rad} \mathrm{s}^{-1}$. Computations simulate transient motions of the rotor system during its acceleration to nominal speed $\Omega=$ $500 \mathrm{rad} \mathrm{s}^{-1}$.

As confirmed by simulations, in the frequency range after the first critical speed, rolls seek positions such as to partially compensate unbalanced forces and decrease vibrations. The "extent" of compensation depends on the axial position of device planes, but for a reasonable selection of planes, a decrease of vibrations looks quite essential in comparison with such of a rotor without autobalancing device.

An important characteristic of the autobalancing process is the speed of roll synchronization. Simulations show that if rotor speed is constant, rolls sooner or later find their stable positions, whereas if the rotor accelerates (or decelerates), the rolls' behavior is different for the different types of device. A one-plane autobalancing device composed of two rolls has proven the most capable of synchronizing within the considered region of rotor speeds. The rolls of such devices are able to find their compensation position and keep it, even when the acceleration of rotor is not very small. Rolls in two-plane devices or one-plane devices comprised of more than two rolls, have difficulties with synchronization under the slightest acceleration.

Some simulation results demonstrating the process of partial unbalance compensation and the difference between oneplane and two-plane devices are presented in Figures 3-15. Figures 3-5 depict the speed of the rotor and the rolls, whereas Figures 6 and 7 show roll angular positions. From the very start, the roll speed stays behind the speed of the rotor. As soon as the acceleration of the rotor is small enough for synchronization, the rolls find their stable positions. As demonstrated in the figures, synchronization is much easier for the one-plane device with two rolls compared to the two-plane device.

Figures 8-11 present the unbalanced force and moment and rotor vibrations in comparison with those of the rotor without autobalancing device. As can be seen, both autobalancing devices efficiently decrease the unbalanced force. On the other hand, the unbalanced moment may even increase. However, the increase of the moment does not diminish the most important

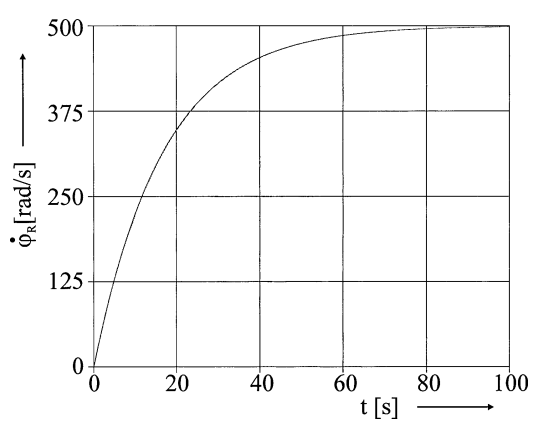

FIGURE 3

Rotor speed. 


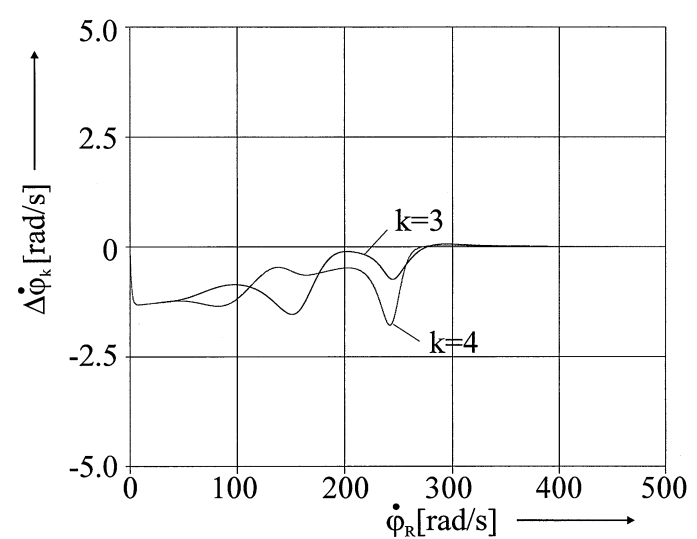

FIGURE 4

One-plane device with two rolls. Difference between rotor and roll speed.

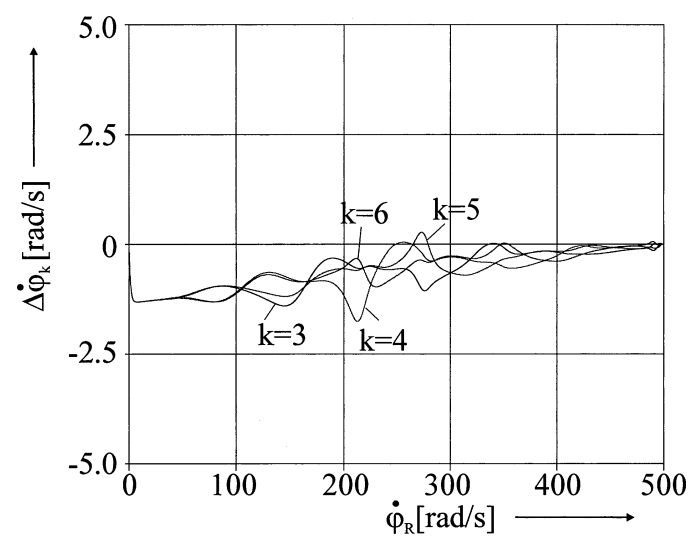

FIGURE 5

Two-plane device with four rolls. Difference between rotor and roll speed.

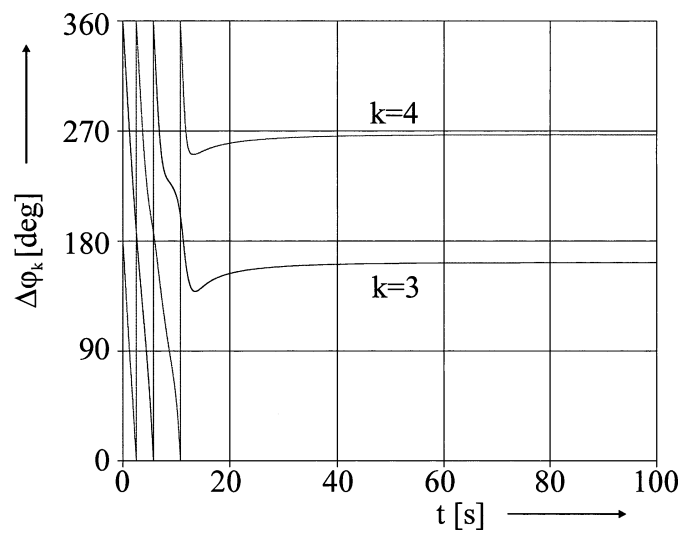

FIGURE 6

One-plane device with two rolls. Roll angular positions.

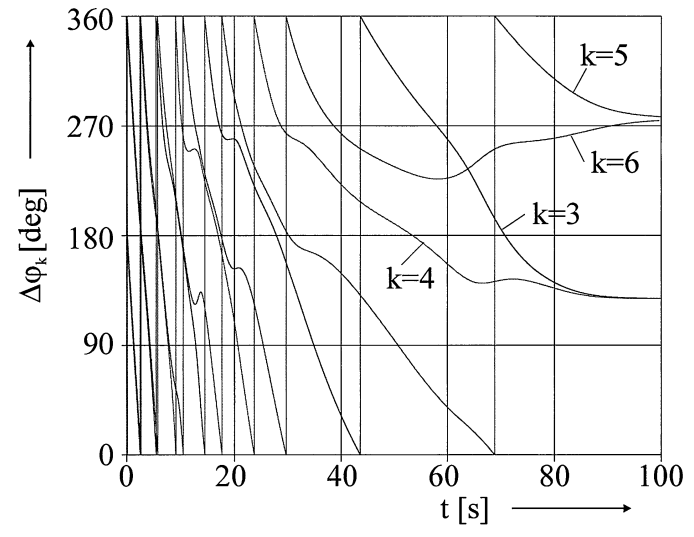

FIGURE 7

Two-plane device with four rolls. Roll angular positions.

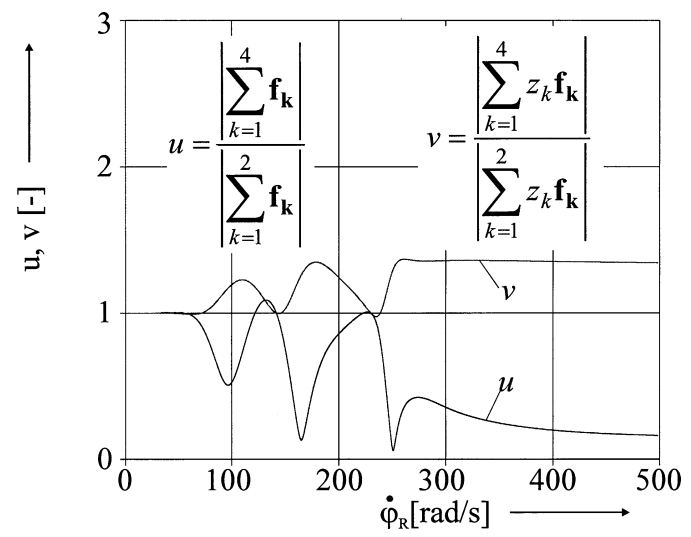

FIGURE 8

One-plane device with two rolls. Unbalanced force and moment.

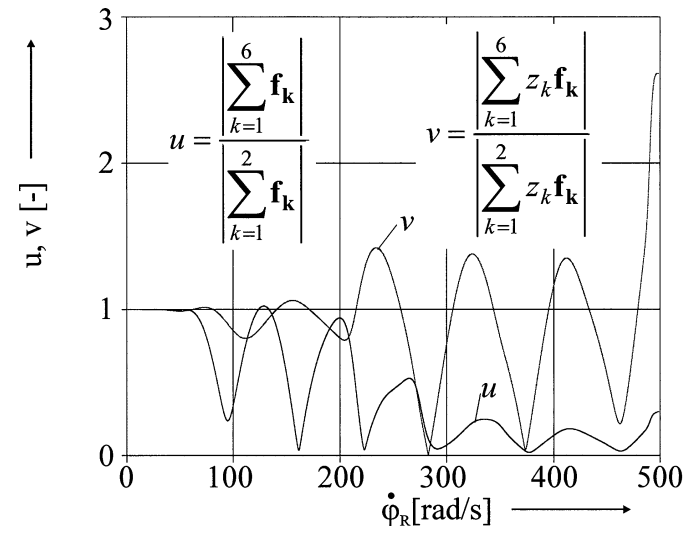

FIGURE 9

Two-plane device with four rolls. Unbalanced force and moment. 


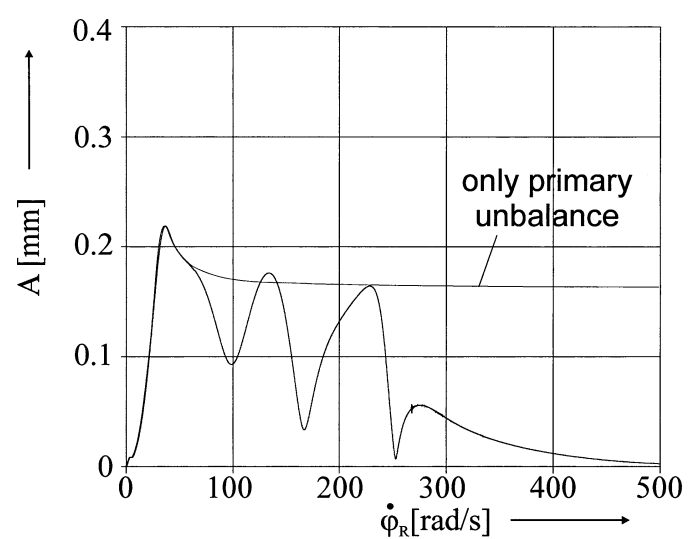

FIGURE 10

One-plane device with two rolls. Amplitude of vibration of rotor top.

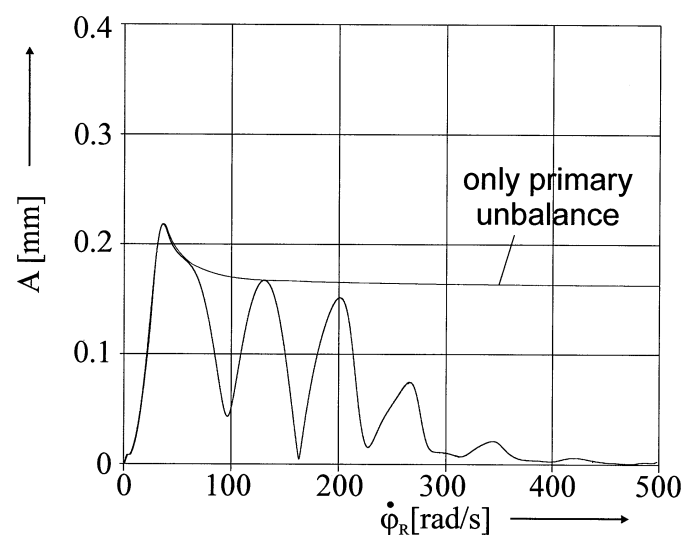

FIGURE 11

Two-plane device with four rolls. Amplitude of vibration of rotor top.

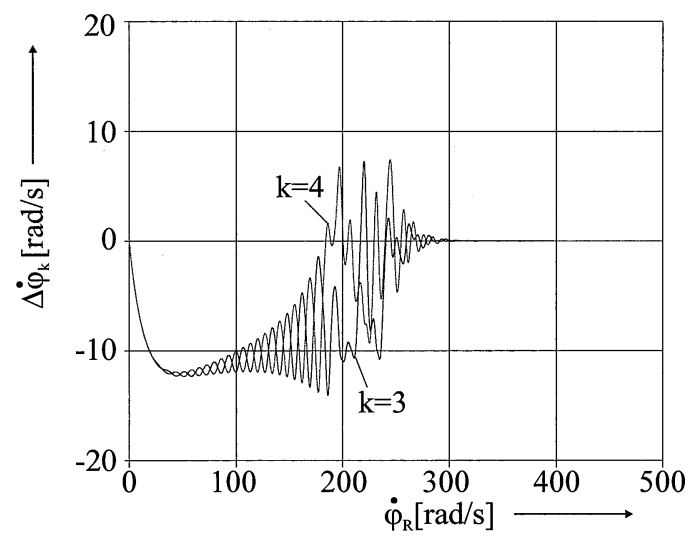

FIGURE 12

One-plane device with two rolls. Low damping. Difference between rotor and roll speeds.

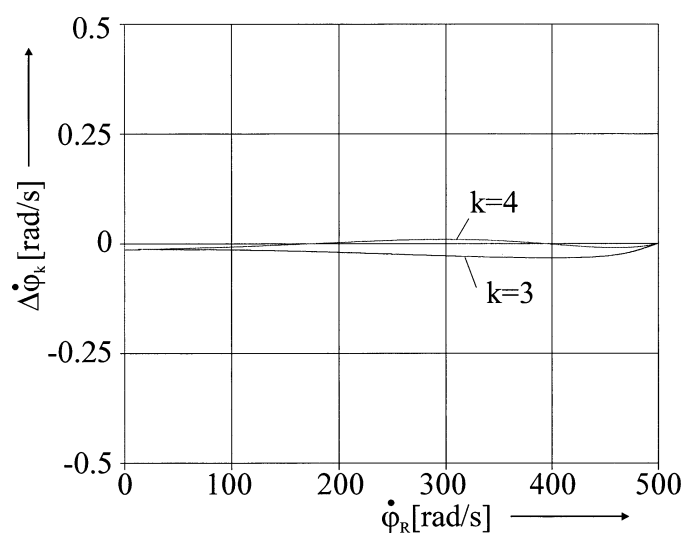

FIGURE 13

One-plane device with two rolls. High damping. Difference between rotor and roll speeds.

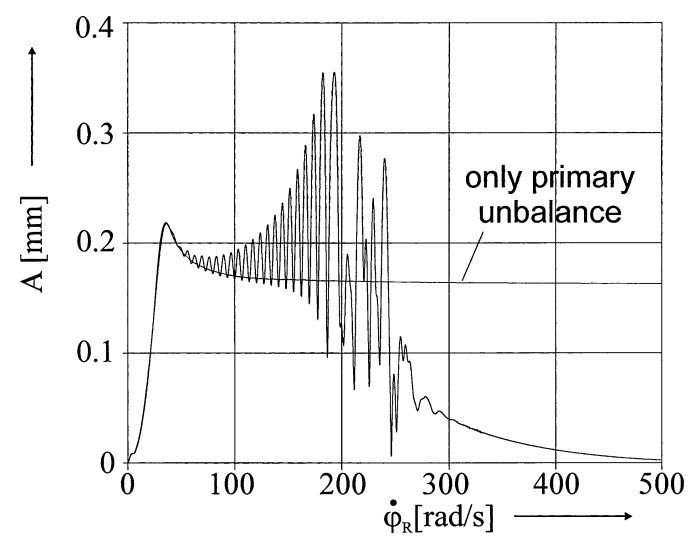

FIGURE 14

One-plane device with two rolls. Low damping. Amplitude of vibration of rotor top.

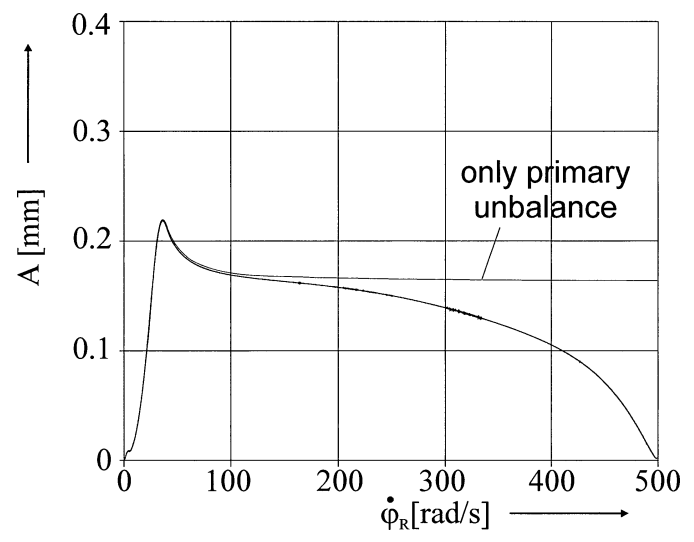

FIGURE 15

One-plane device with two rolls. High damping. Amplitude of vibration of rotor top. 
effect provided by the autobalancing device, i.e., the essential decrease of vibrations.

The character of transient processes sufficiently depends on the value of damping parameters $\beta_{i}$. Figures 12-15 show the difference between high and low-level damping for a one-plane device with two rolls.

For low damping, rolls, when passing through the critical speed, may come to the Sommerfeld-type motion (Sperling et al., 2001, 2002), i.e., they keep on moving at the speed, equal to the critical speed, when the rotor accelerates and leaves this speed region. The Sommerfeld-type motion engenders increased vibrations of the rotor near critical speed. Roll synchronization in the case of low damping is accompanied by oscillations, hampering the process (Figures 12 and 14).

When damping is comparatively high, the difference between the rotor speed and the roll speed remains rather low during acceleration, but synchronization starts later and takes more time compared to the system with lower values of $\beta_{i}$ (Figures 13 and 15 ). As computations show, for the rotor system under consideration it is possible to find an optimal damping level providing good synchronization without any noticeable occurrence of the Sommerfeld-effect near critical speed.

\section{CONCLUSION}

Analytical investigations supported by results of computer simulations prove that under certain conditions autobalancing devices can provide partial compensation of unbalance and essential decrease of vibrations in the speed range after the first critical speed. The possibility of partial unbalance compensation sufficiently extends the field of potential applications of autobalancing devices. Although the present publication is restricted to consideration of the rigid rotor autobalancing, our recent investigations show that partial compensation of unbalance under certain conditions may be achieved for flexible rotors.

Among the most important practical applications of autobalancing devices are centrifuges, hand power tools, and washing machines. Lately, the information of using autobalancing devices in optical disk drives has appeared (Kang et al., 2001; Huang and Chao, 2002).

Authors intend to continue their investigations of autobalancing phenomena, and focus on the effect of partial unbalance compensation as well as on the problems of optimal choice of parameters.

\section{ACKNOWLEDGEMENTS}

The authors would like to express gratitude to the Deutsche Forschungsgemeinschaft for their financial support (No.Sp $462 / 7-1)$

\section{REFERENCES}

Blekhman, I. 1976. Method of direct motion separation in problems of vibration acting on nonlinear mechanical systems. Izv. AN SSSR Mechanika Tverdogo Tela 11(6):13-27.

Blekhman, I. 2000. Vibrational Mechanics. World Scientific, Singapore, 509.

Bövik, P., and Högfors, C. 1986. Autobalancing of rotors. Journal of Sound and Vibration 111(3):429-440.

Chung, J., and Ro, D. S. 1999. Dynamical analysis of an automatic dynamic balancer for rotating mechanisms. Journal of Sound and Vibration 228(5):1035-1056.

Hedaya, M., and Sharp, R. 1977. An analysis of a new type of automatic balancer. Journal Mechanical Engineering Science 19(5):221-226.

Huang, W. Y., and Chao, C. P. 2002. The application of ball-type balancers for radial vibration reduction of high speed optic drives. Journal of Sound and Vibration 250(3):415-430.

Kang, J. R., Chao, C. P., Huang, C. L., and Sung, C. K. 2001. The dynamics of a ball-type balancer system equipped with a pair of freemoving balancing masses. Transactions of the ASME 123(10):456465.

Kapiza, P. 1951. Dynamic stability of a pendulum with vibrating support point. Journal Experimental and Theoretical Physics 21(5):588-598.

Sperling, L., Merten, F., and Duckstein, H. 1998. Analytical and numerical investigations of rotation-vibration-phenomena. Nonlinear Oscillations in Mechanical Systems, Proceedings of the XXV-XXVI Summer Schools, St. Petersburg, Russia (1):145-159.

Sperling, L., and Duckstein, H. 2001. Zum selbsttätigen Auswuchten des starren Rotors in zwei Ebenen, in Irretier, H., Nordmann, R., and Springer, H. (Eds.), SIRM 2001 Schwingungen in rotierenden Maschinen $V$, Wien. Friedr. Vieweg \& Sohn Verlagsgesellschaft mbH Braunschweig-Wiesbaden, 161-168.

Sperling, L., Ryzhik, B., and Duckstein, H. 2001. Two-plane automatic balancing, Machine Dynamics Problems, Proceedings of 7th PolishGerman Workshop on Dynamical Problems in Mechanical Systems 139-152.

Sperling, L., Ryzhik, B., Linz, C., and Duckstein, H. 2002. Simulation of two-plane automatic balancing of a rigid rotor. Mathematics and Computers in Simulation 58:351-365.

Thearle, E. 1932. A new type of dynamic-balancing machine. Transactions of the ASME 54(12):131-141. 

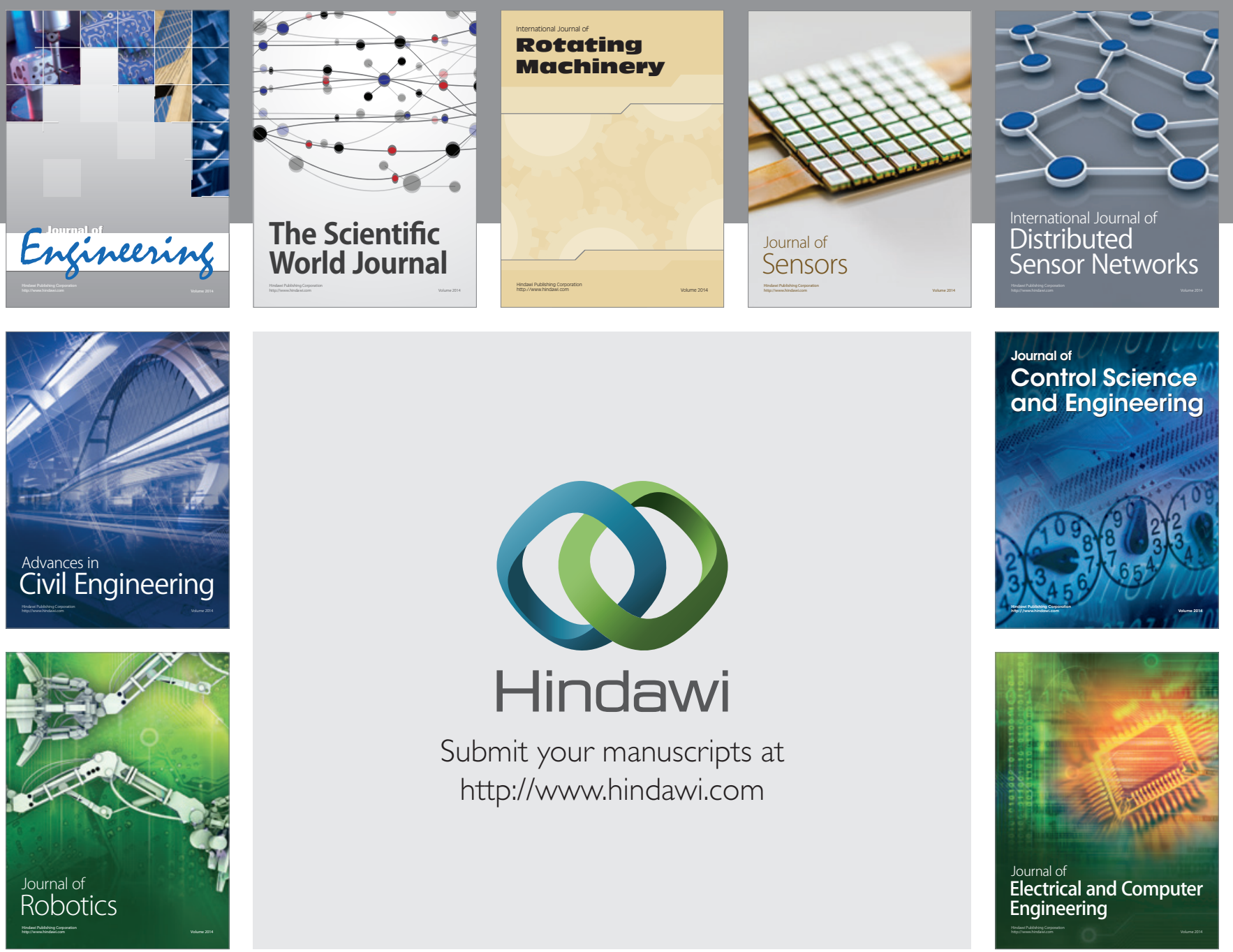

Submit your manuscripts at

http://www.hindawi.com
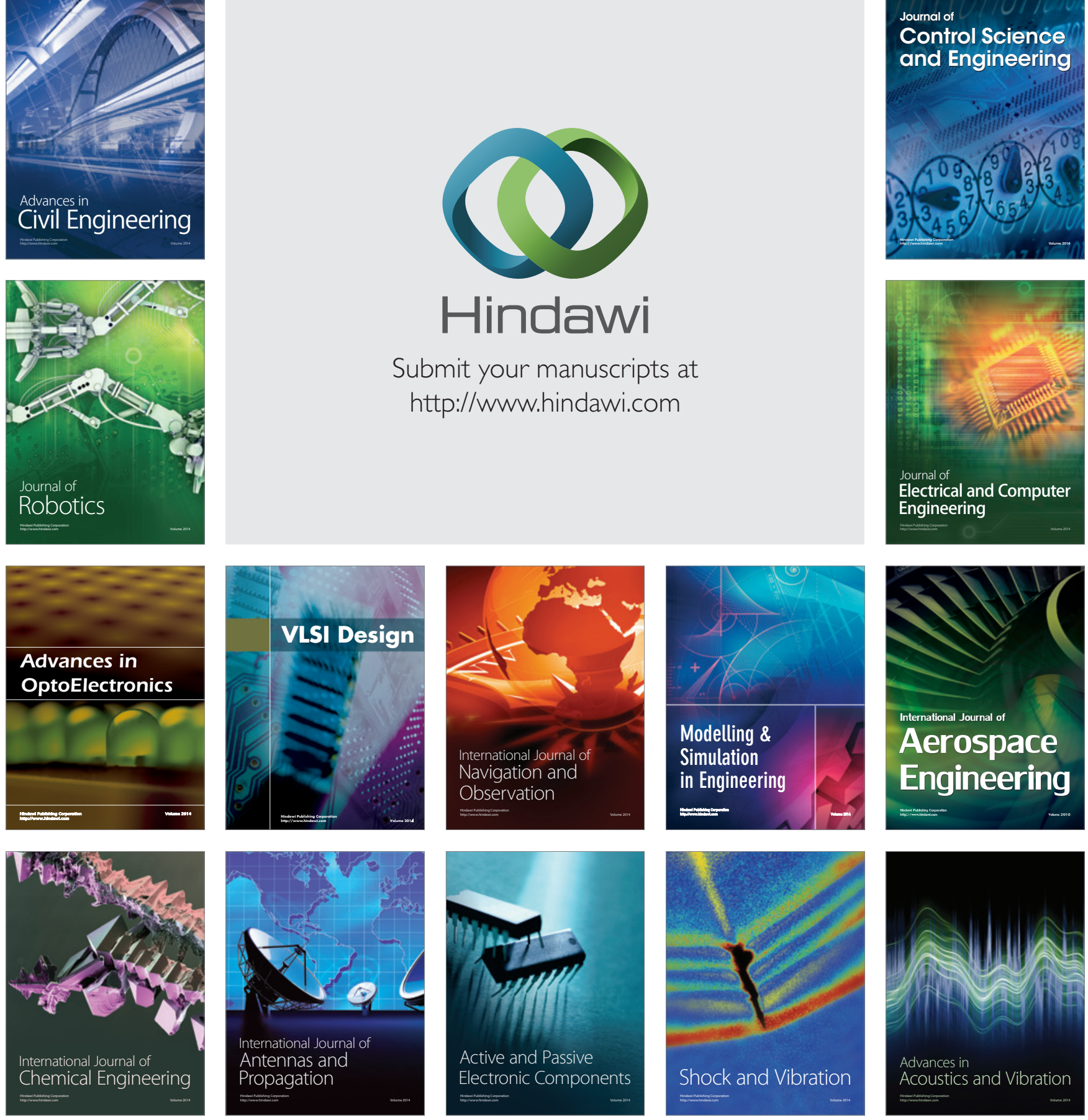